Livraisons

d'Histoire

de l'Architecture

\section{Livraisons de l'histoire de l'architecture}

39 | 2020

Maquettes d'architecture

\title{
Alphonse Goutès (1839-1898), architecte et professeur à l'École régionale des beaux-arts de Montpellier
}

Alphonse Goutès (1839-1898), architect and professor at the École régionale des beaux-arts de Montpellier

Alphonse Goutès (1839-1898), Architekt und Professor an der École régionale des Beaux-Arts von Montpellier

Théodore Guuinic

\section{OpenEdition}

Journals

Édition électronique

URL : https://journals.openedition.org//ha/1612

DOI : 10.4000/lha.1612

ISSN : 1960-5994

Éditeur

Association Livraisons d'histoire de l'architecture - LHA

Édition imprimée

Date de publication : 15 juin 2020

Pagination : $127-140$

ISSN : 1627-4970

Référence électronique

Théodore Guuinic, « Alphonse Goutès (1839-1898), architecte et professeur à l'École régionale des beaux-arts de Montpellier », Livraisons de l'histoire de l'architecture [En ligne], 39 | 2020, mis en ligne le 24 janvier 2021, consulté le 28 juin 2022. URL : http://journals.openedition.org/lha/1612 ; DOI : https:// doi.org/10.4000/lha.1612 
Varia 



\section{ALPHONSE GOUTÈS (1839-1898), ARCHITECTE ET PROFESSEUR À L'ÉCOLE RÉGIONALE DES BEAUX-ARTS DE MONTPELLIER}

À la fin du XIX siècle, la ville de Montpellier offre un enseignement de l'architecture depuis déjà plus d'un siècle. Dispensé au sein de plusieurs établissements successifs depuis la fin de l'Ancien Régime, cet enseignement fait l'objet d'une ambitieuse restructuration sous la III ${ }^{e}$ République, dans le cadre de l'École des beaux-arts de Montpellier. L'État assigne alors à l'établissement une vocation et un rayonnement nouveaux, en l'érigeant en École régionale des beaux-arts. Celle-ci connaît à cette époque une diversification des enseignements, apportant aux élèves une formation approfondie, dans le sillage des réformes pédagogiques parisiennes. Pourtant, en dépit de progrès notables, Montpellier ne figurera pas dans la liste des villes choisies pour accueillir l'une des Écoles régionales d'architecture instituées par le décret de $1903^{1}$. C'est donc au sein de la section d'architecture de l'École régionale des beaux-arts que l'enseignement de l'architecture persiste ensuite à Montpellier. À l'heure où l'on se penche sur l'histoire des enseignements de l'architecture au $\mathrm{XX}^{\mathrm{e}}$ siècle ${ }^{2}$, il convient donc, pour le cas montpelliérain, d'étudier sa mise en place à la fin du siècle précédent. Dans cette optique, nous tenterons d'esquisser ici, en l'état actuel de nos connaissances ${ }^{3}$, la construction d'un enseignement régional contemporain du débat sur les Écoles régionales d'architecture ${ }^{4}$, à travers la figure de l'architecte et professeur Alphonse Goutès.

1. Décret ministériel du 23 janvier 1903 et arrêté du 26 janvier 1903, Journal officiel de la République française, 31 janvier 1903.

2. Cet article est le fruit d'une recherche engagée dans le cadre du programme HEnsA20 du ministère de la Culture et d'une communication exposée lors du $5^{\mathrm{e}}$ séminaire qui s'est tenu les 30 novembre et $1^{\text {er }}$ décembre 2018 au sein des Écoles nationales supérieures d'architecture de Marseille et de Montpellier.

3. Ces recherches s'inscrivent dans le cadre de notre thèse en cours, "Faire école : héritages bâtis et réinvention des modèles entre Paris et le Midi méditerranéen (XVIII $-\mathrm{XX}^{\mathrm{e}} \mathrm{s}$.) ", thèse en Architecture sous la direction de Thierry Verdier et Frédérique Villemur, ENSA de Montpellier et Université Paul Valéry-Montpellier III.

4. Jean-Pierre Epron, Éclectisme et profession, la création des Écoles régionales 1889-1903, Rapport de recherche pour le Bureau de la recherche architecturale - Direction de l'architecture et de l'urbanisme, $1987,431 \mathrm{p}$. 


\section{Des sources renouvelées}

Les archives de l'École des beaux-arts de Montpellier relatives à cette époque sont abondantes, mais incomplètes et inégales ${ }^{5}$. L'absence de travaux d'élèvesarchitectes constitue, d'une part, une perte irréparable; d'autre part, les activités de l'école sont de moins en moins documentées au long de son histoire, en particulier au cours du $\mathrm{XX}^{\mathrm{e}}$ siècle. Les rares travaux entrepris sur ce terrain reflètent d'ailleurs cette disparité des sources, et concentrent leur attention sur la vitalité de l'école au tournant des $\mathrm{XIX}^{\mathrm{e}}$ et $\mathrm{XX}^{\mathrm{e}}$ siècles, à l'image de la thèse de Dominique Laredo ${ }^{6}$. Bénéficiant de tels acquis, une histoire de la section d'architecture de l'école peut aujourd'hui apporter de nouveaux éclairages en s'appuyant sur des archives encore inexploitées. Parmi elles, l'ensemble des manuscrits de cours légués par l'architecte et professeur Alphonse Goutès ${ }^{7}$ constitue une pièce maîtresse pour apprécier l'enseignement de l'architecture dispensé à cette époque. S'il est encore trop tôt pour en livrer une lecture exhaustive, on peut néanmoins tenter d'en esquisser les traits généraux, qui suggèrent l'originalité du professorat d'Alphonse Goutès. Pour cela, il est d'abord nécessaire de le replacer dans l'histoire au long cours de l'enseignement montpelliérain, dont certaines permanences marquent la vocation de l'école sur près d'un siècle d'existence.

\section{Les lieux de l'enseignement de l'architecture à Montpellier (1779-1872)}

À Montpellier, la première école proprement dite à offrir des cours d'architecture est celle de la Société des Beaux-Arts, fondée en 1779, où les architectes JeanAntoine Giral, Jacques Donnat et Charles Durand dispensent un cours d'architecture en prolongement des cours gratuits de l'école de dessin. L'établissement, qui occupe l'ancien collège des jésuites, est ensuite intégré en 1787 à l'École des Ponts et chaussées de la ville ${ }^{8}$, où les cours d'architecture s'appuient principalement sur

5. Ces archives sont principalement conservées aux Archives municipales de Montpellier, aux Archives départementales de l'Hérault, à la Bibliothèque municipale de Montpellier, ainsi qu'aux Archives nationales.

6. Dominique Laredo, "La vie artistique à Montpellier de 1870 à 1918 ", thèse de doctorat sous la direction de Laure Pellicer, univ. Paul Valéry-Montpellier III, 1991, 1062 p. Ce travail, particulièrement méritoire pour son étendue, constitue le principal apport à la connaissance de la vie artistique à Montpellier sous la III ${ }^{\mathrm{e}}$ République, et en particulier à l'histoire de l'École des beaux-arts de la ville à cette époque (op. cit., p. 20-133). Sur cette même période, voir également Bernard Derrieu, «L'École des beaux-arts de Montpellier et la formation des sculpteurs sous la Troisième République », sous-direction de l'Inventaire Général, Direction régionale des affaires culturelles Languedoc-Roussillon, 1993.

7. Alphonse Goutès, manuscrits des cours dispensés à l'École régionale des beaux-arts de Montpellier, (désormais Cours d'architecture), Bibliothèque municipale de Montpellier, Fonds patrimoniaux, Magasin Cavalier, 25 vol., in-8 $8^{\circ}$ MS0244.

8. Théodore Guuinic, "Apprendre l'art de bâtir à Montpellier à la veille de la Révolution, à travers le Cours inédit de Charles Durand (1791) ", communication au III ${ }^{\mathrm{e}}$ Congrès francophone d'histoire de la construction, Nantes, 21-23 juin 2017, dans Gilles Bienvenu, Martial Monteil et Hélène Rousteau-Chambon (dir.), Construire! Entre Antiquité et époque contemporaine, Paris, Éditions A\&J Picard, 2019, 1314 p., p. 185-197. 
le commentaire de traités et recueils parisiens ${ }^{9}$. Si la continuité d'un enseignement du dessin est assurée à l'École centrale en 1796, les cours d'architecture s'interrompent sous la Terreur et se réduiront alors à l'enseignement particulier et éphémère de Claude-Mathieu Delagardette ${ }^{10}$. Il faut ensuite attendre quelques années avant que la trace d'un professeur d'architecture ne réapparaisse au sein de l'école municipale, refondée en 1806. Exceptée cette courte interruption, l'architecture semble ainsi avoir été enseignée continûment à Montpellier jusqu'au $\mathrm{XX}^{\mathrm{e}}$ siècle, la carrière de certains professeurs, tels Joseph Boué et Louis Corvetto, s'étendant sur près de cinq décennies ${ }^{11}$. Pourtant, une attention aux lieux de ces enseignements trahit une trajectoire moins linéaire. Au cours de cette histoire, les cours d'architecture se tiennent en trois lieux principaux : d'abord dispensés dans l'ancien collège des jésuites, ils s'établissent ensuite dans des locaux exigus de l'Hôtel de Ville, place de la Canourgue ; ils doivent par la suite déménager à nouveau avant de se réinstaller enfin vers 1840 boulevard de l'Esplanade, dans de nouveaux locaux attenants au Musée Fabre (ill. 1), qu'ils ne quitteront pas avant 1968.

Sur cette période, la vocation des cours semble à première vue assez constante : celle d'une école gratuite d'apprentissage, où se côtoient artistes et artisans, dans la tradition des écoles gratuites de dessin des Lumières. Au cours du XIX ${ }^{\mathrm{e}}$ siècle s'opère cependant une transition progressive d'un enseignement académique conçu autour du dessin à un enseignement des trois arts, attentif à l'essor de l'industrie et au contexte économique local. Dans les années 1860, les classes de dessin d'ornement et de dessin industriel prennent ainsi de l'importance au point d'annexer le cours d'architecture, tandis que les cours de dessin, sculpture et de stéréotomie sont déplacés pour s'adapter aux disponibilités des apprentis et ouvriers ${ }^{12}$.

\section{La refondation de l'École des beaux-arts de Montpellier (1871-1882)}

Au lendemain de la guerre de 1870, l'école va bénéficier d'un nouveau souffle favorisé par la politique ambitieuse de la IIIe République en matière d'instruction. Comme l'a bien montré Dominique Laredo ${ }^{13}$, le renouveau de l'établissement doit beaucoup à son nouveau directeur, Ernest Michel. Lauréat du premier Grand prix

9. Théodore Guuinic, "L'influence de J.-F. Blondel sur le cours d'architecture de l'école des Ponts et chaussées de Montpellier", communication au colloque "Jacques-François Blondel et l'enseignement de l'architecture. La dernière leçon de l'architecture “à la française” ", décembre 2017, Cité de l'architecture et du patrimoine, Paris, à paraître.

10. Avant son départ de Montpellier et sa mort prématurée en 1805, Claude-Mathieu Delagardette (1862-1805) a notamment formé le jeune Jean-Joseph Boué (1784-1868).

11. Arch. mun. de Montpellier, R1/9 (104) et R112. La chronique de l'enseignement artistique montpelliérain au cours de ces décennies a été établie par Coraline Manches, dans «Les écoles d'art de Montpellier de 1806 à 1870 ", Mémoire de maîtrise d'histoire de l'art, dir. Odile Foucaud, Université Paul Valéry-Montpellier III, 2000, 135 p.

12. Ibid.

13. Dominique Laredo, op. cit., p. 26 sq. 


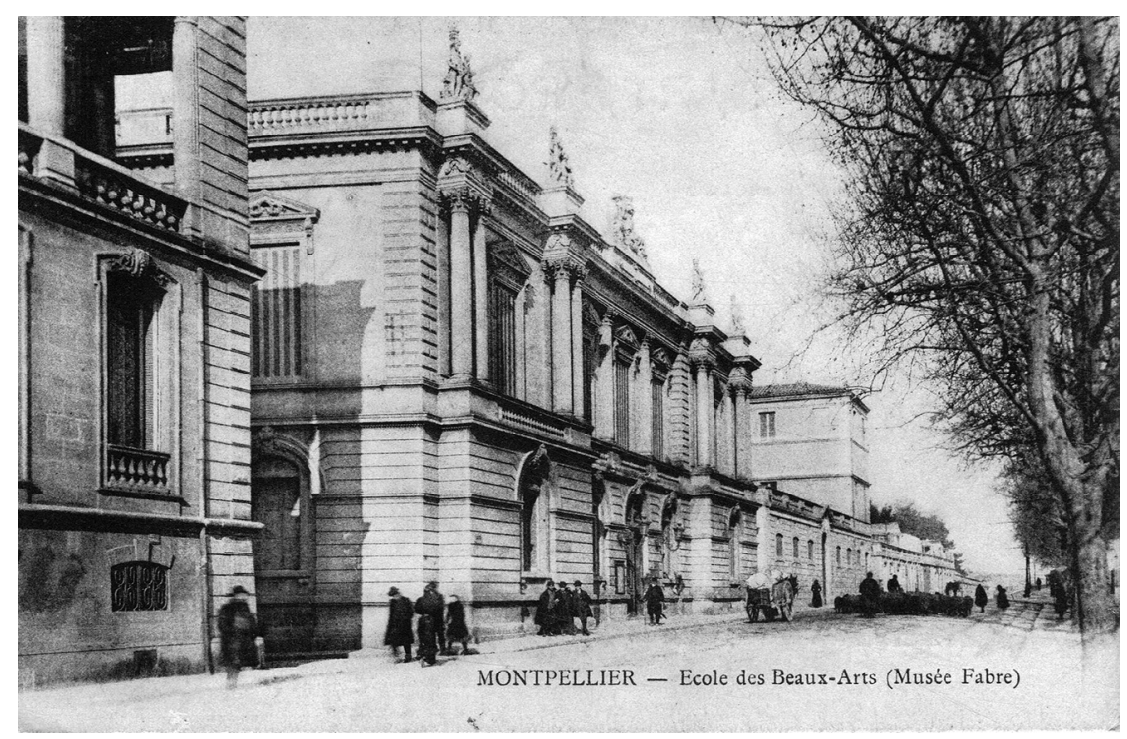

Ill. 1 : L'École régionale des beaux-arts de Montpellier, début du XXe siècle, photographie anonyme (carte postale).

de Rome de peinture en 1860, Ernest Michel restera directeur de l'École des beaux-arts durant trente ans, de 1871 à 1901. Au cours de ces trois décennies, l'école connaîtra grâce à lui des évolutions significatives, dans le programme pédagogique comme dans la composition du corps enseignant. Ainsi s'ouvrent en 1878 de nouveaux cours d'histoire de l'art et de perspective, tandis qu'un second professeur de dessin est recruté. Un an plus tard, l'État ouvre un concours d'enseignement du dessin et instaure un certificat d'aptitude ${ }^{14}$ ouvrant de nouveaux débouchés aux élèves de l'école. Au même moment, l'établissement adopte un nouveau règlement ${ }^{15}$ pour encadrer le séjour des élèves boursiers poursuivant leur formation à l'École des beaux-arts de Paris, dont le nombre est croissant depuis 1850. L'école compte alors environ 260 élèves, pour 8 professeurs ${ }^{16}$.

C'est dans ce contexte privilégié que, soutenue par une politique volontariste en matière d'instruction publique illustrée par les lois Ferry, l'école signe avec l'État et la ville de Montpellier une convention ${ }^{17}$ lui conférant un rayonnement régional.

14. Rapport et arrêté sur l'enseignement du dessin dans les établissements publics, 24 mars 1879; Décret relatif au concours pour le certificat d'aptitude à enseigner le dessin, 6 août 1880 ; circulaire du ministère du 19 novembre 1880 .

15. Règlement des pensionnaires de la ville à l'École nationale des beaux-arts de Paris, 13 décembre 1880, Montpellier, Cristin et $\mathrm{C}^{\mathrm{ie}}$, AM de Montpellier, R1/9 (113).

16. Arch. mun. de Montpellier, R1/9 (111).

17. Arch. mun. de Montpellier, R1/9 (104), Convention entre le Ministre de l'Instruction Publique et le Maire de Montpellier, 11 mars 1882, transformant l'École Municipale des Beaux-Arts de Montpellier en École Régionale des Beaux-Arts de Montpellier, Montpellier, Impr. Cristin, Serre et Ricome, 1882, $12 \mathrm{p}$. 
Cette convention paraît marquer un tournant dans l'histoire de l'école, notamment parce qu'elle entérine le soutien financier de l'État à hauteur du quart du budget de fonctionnement de l'école (4000 francs). En contrepartie, l'école dépend désormais de l'agrément de l'administration des Beaux-Arts pour toute modification concernant le budget de l'établissement ${ }^{18}$ et les traitements des professeurs. Cet accord est doublement avantageux pour l'école, car la nomination du directeur et des enseignants reste à la discrétion du maire de la ville et du préfet de l'Hérault : l'école peut dès lors se doter d'un programme pédagogique plus complet, tout en restant relativement indépendante de l'administration des Beaux-Arts pour le choix des professeurs et l'élaboration de ses enseignements. En 1888, le programme pédagogique $^{19}$ mentionnera ainsi neuf enseignants pour quatorze disciplines enseignées, dont quatre relatives à l'architecture, dispensées par l'architecte Alphonse Goutès.

\section{Un professeur d'architecture aux talents multiples}

Pour apprécier précisément la pédagogie de la section d'architecture à cette époque, la figure d'Alphonse Goutès apparaît comme centrale, l'architecte dispensant jusqu'à sept cours différents ${ }^{20}$ au cours de ces années. Fils de plâtrier, Goutès naît en 1839 à Montpellier où il débute à l'école municipale de dessin. Il achève ensuite sa formation à Paris à l'École impériale et spéciale des beaux-arts ${ }^{21}$ dans l'atelier de Charles-Auguste Questel où il étudie entre 1860 et 1863, assistant probablement aux cours controversés de Viollet-le-Duc à la suite de la réforme de l'enseignement des Beaux-Arts. Faute de disposer d'un portrait de l'architecte, quelques éléments biographiques livrés par ses manuscrits permettent d'en suggérer les contours. Amateur de poésie occitane et dramaturge à ses heures, Goutès possède également un talent d'aquarelliste, couronné par une participation à une dizaine de salons à Montpellier et à Paris. À Montpellier, Goutès vécut dans l'écusson, rue du Logis Saint-Paul. L'histoire réserve ici un détail symbolique : face à lui s'élève un immeuble construit sous la Révolution par Charles Durand, son prédécesseur au poste de professeur d'architecture à l'école des Ponts et chaussées, un siècle plus tôt. Comme lui ancien élève de l'école municipale de dessin, Goutès s'inscrit dans la lignée de plusieurs enfants du pays qui, achevant leur formation à la capitale, reviennent à Montpellier et deviennent à leur tour les passeurs ${ }^{22}$ d'un héritage enrichi au contact des écoles parisiennes.

18. Convention de 1882, op. cit., articles 2 et 5.

19. Programme de l'enseignement, École régionale des beaux-arts de Montpellier, Montpellier, Serre \& Ricome, 1888, 29 p.

20. Ibid., p. 11-15. Perspective, classes préparatoire et élémentaire de dessin géométrique, classe de dessin industriel, classe d'ornement, classe et atelier d'architecture, et classe de composition décorative.

21. Arch. nat., $\mathrm{AJ}^{52} 367$.

22. Une lignée composée de J.-R. Demoulin, J. Boué, Ch. Abric, A. Goutès et qui sera prolongée par ses élèves. 


\section{Son auvre d'architecte}

Si Goutès se distingue par sa carrière professorale, on lui doit également plusieurs réalisations comme architecte et restaurateur. Son œuvre d'architecte, concentré à Montpellier, se compose de trois bâtiments construits et d'un projet non réalisé. Goutès revient d'abord à Montpellier en 1870 pour la construction d'une chapelle funéraire dans le cimetière Saint-Lazare. Quelques années plus tard, il s'installe à Montpellier et se voit chargé de la restauration et du réaménagement de la Tour des Pins, monument emblématique de la ville appartenant à l'enceinte défensive du XII ${ }^{e}$ siècle. Goutès restaure l'édifice et en conçoit un nouvel aménagement afin d'y accueillir les archives municipales. Nommé architecte de la ville en avril 1881, il laisse alors un projet remarqué dans le cadre du concours pour la reconstruction de la Comédie : son projet ${ }^{23}$, intégrant une machinerie de fer, s'inspire de la façade composée par Garnier pour l'opéra de Paris (ill. 2). Le jury souligne l'élégance du projet et la compétence de l'architecte mais le classe néanmoins second, derrière le projet finalement réalisé de Cassien-Bernard, lui aussi élève de Questel. En 1885, alors que s'achève le chantier de la Tour des Pins, Goutès est chargé de construire le lycée de jeunes filles de Montpellier ${ }^{24}$ (actuel lycée Clemenceau). L'édifice, conçu pour accueillir une centaine d'élèves, est le premier lycée de jeunes filles de France et sera inauguré par le président de la République Sadi Carnot en mai 1890. Entre-temps, Goutès signe également l'aménagement d'un parc paysager sur la motte castrale de Mauguio ${ }^{25}$, pour lequel il conçoit, autour d'une tour-belvédère, une composition d'allées concentriques garnies de buis, d'essences exotiques et d'une grotte en rocaille.

Malgré sa variété, l'œuvre construit de Goutès demeure relativement modeste et restera dans l'ombre de celle de son contemporain et ami d'enfance Léopold Carlier, qu'il avait suivi dans l'atelier Questel. C'est donc principalement comme enseignant que Goutès marque l'histoire de la ville, et qu'il jouit à la fin de sa vie d'une notoriété importante à l'échelle de la région, où il a acquis la reconnaissance de ses pairs. Parallèlement à sa fonction de professeur, il est alors membre du conseil des bâtiments civils du département et vice-président de la Société des architectes du Midi de la France.

23. Projet de théâtre pour la ville de Montpellier, vue perspective, Arch. mun. de Montpellier, 3Fi9; Idem, élévations des façades avant et arrière du théâtre, 3Fi28a \& 3Fi28b. Je remercie Olivier Liardet d'avoir attiré mon attention sur ces dessins.

24. Édifié suite à la loi Camille Sée (1880), le bâtiment s’élève sur deux étages; de plan rectangulaire, il dispose les salles de classes autour d'une cour et d'un jardin planté d'essences rares.

25. Relief artificiel réalisé au $\mathrm{X}^{\mathrm{e}}$ siècle, la motte de Mauguio est, par ses dimensions, l'une des plus importantes du Sud de la France. Les plantations seront effectuées après la mort de Goutès par l'un de ses élèves, Henri Michel, qui sera chargé d'édifier au centre du jardin un château d'eau (1902-1904). D'abord protégé comme site classé, l'ensemble est aujourd'hui inscrit au titre des monuments historiques et s'est vu attribuer le label Jardin remarquable en 2014 (notes d'Yvon Comte et Olivier Liardet, Direction régionale des affaires culturelles Occitanie). 


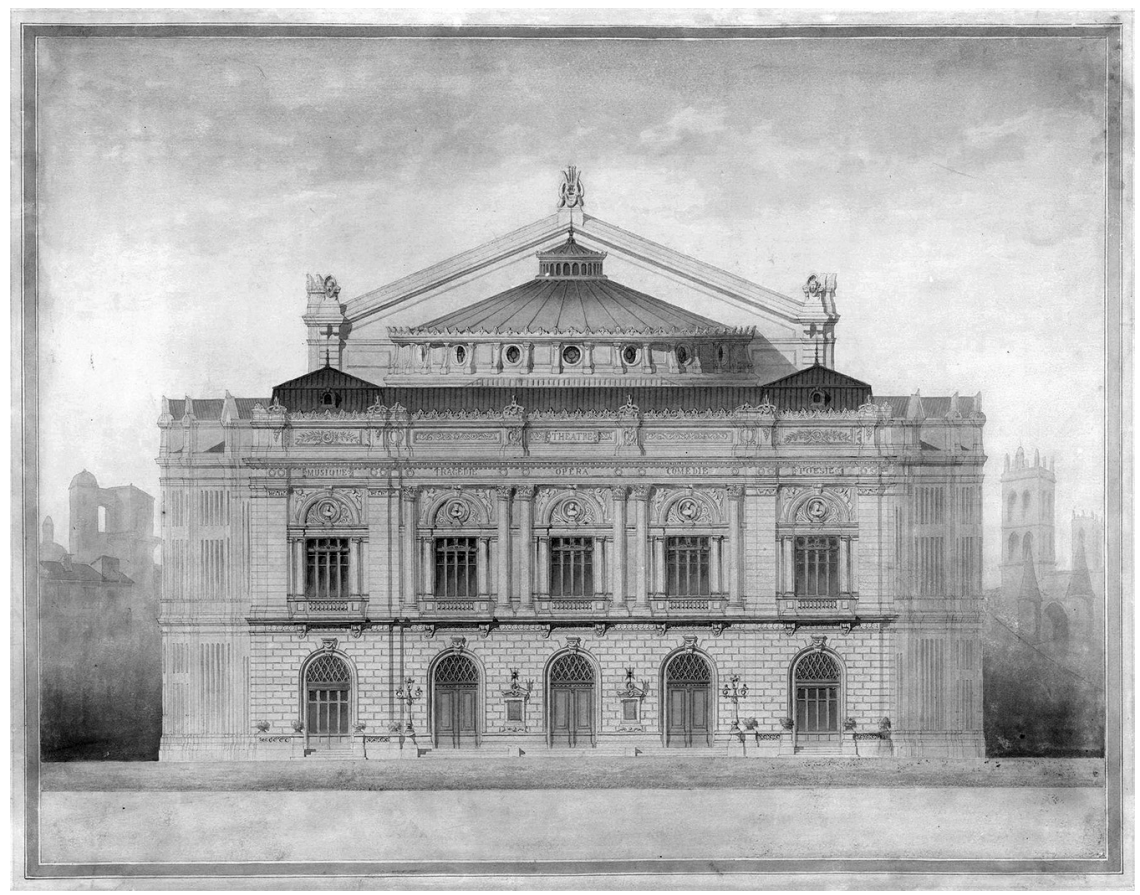

Ill. 2 : Alphonse Goutès, Théâtre de Montpellier, élévation de la façade arrière (1881), Archives Municipales de Montpellier, 3Fi28a. (C) Médiathèque Centrale Émile Zola, Montpellier Méditerranée Métropole.

\section{Le professorat d'Alphonse Goutès, au prisme de ses manuscrits}

Si l'héritage principal de Goutès est à rechercher dans sa carrière de pédagogue, la découverte récente ${ }^{26}$ de ses manuscrits de cours constitue un apport essentiel pour affiner la connaissance de ce personnage méconnu et réévaluer son importance. Les manuscrits légués par Goutès promettent en effet d'offrir un aperçu en profondeur des cours qu'il dispense à l'École des beaux-arts de Montpellier, au long d'un professorat long de deux décennies. Bien qu'il soit encore trop tôt pour mener une analyse approfondie des manuscrits et apprécier finement son approche pédagogique, il est néanmoins possible d'en offrir une première présentation et, avec elle, de formuler quelques hypothèses. À première vue, ces carnets compilent des matériaux très divers : des notes préparatoires de cours, marquées par un caractère d'oralité, mais également des exercices, des programmes d'architecture et même des chansons d'atelier; s'y ajoutent encore des notes de lectures, instructives, et des recueils d'ornements. Ainsi transparaît à travers eux la structure des cours de

26. Je remercie vivement Nathalie Tailleur, des fonds patrimoniaux de la Bibliothèque municipale de Montpellier, d'avoir identifié et de m'avoir permis de consulter ces manuscrits. 
Goutès, bien que la composition des volumes ne coïncide pas exactement avec ses charges d'enseignement, certains contenus excédant le cadre de ses prérogatives.

\section{Les enseignements d'Alphonse Goutès}

Installé à Montpellier depuis 1878, Goutès est d'abord chargé du cours de perspective et géométrie descriptive ${ }^{27}$. Parallèlement, il dispense des cours d'histoire de l'architecture ${ }^{28}$, en synergie avec le professeur d'histoire de l'art, M. Edet. À partir de 1886, Goutès est chargé des cours de dessin géométrique et du cours d'architecture $^{29}$ dans lesquels l'histoire de l'architecture est très présente, selon des modalités qui évoluent au gré de la progression des élèves. Goutès consacre ainsi la $1^{\text {re }}$ année à la découverte des principes et des éléments d'architecture ${ }^{30}$ (ill. 3),

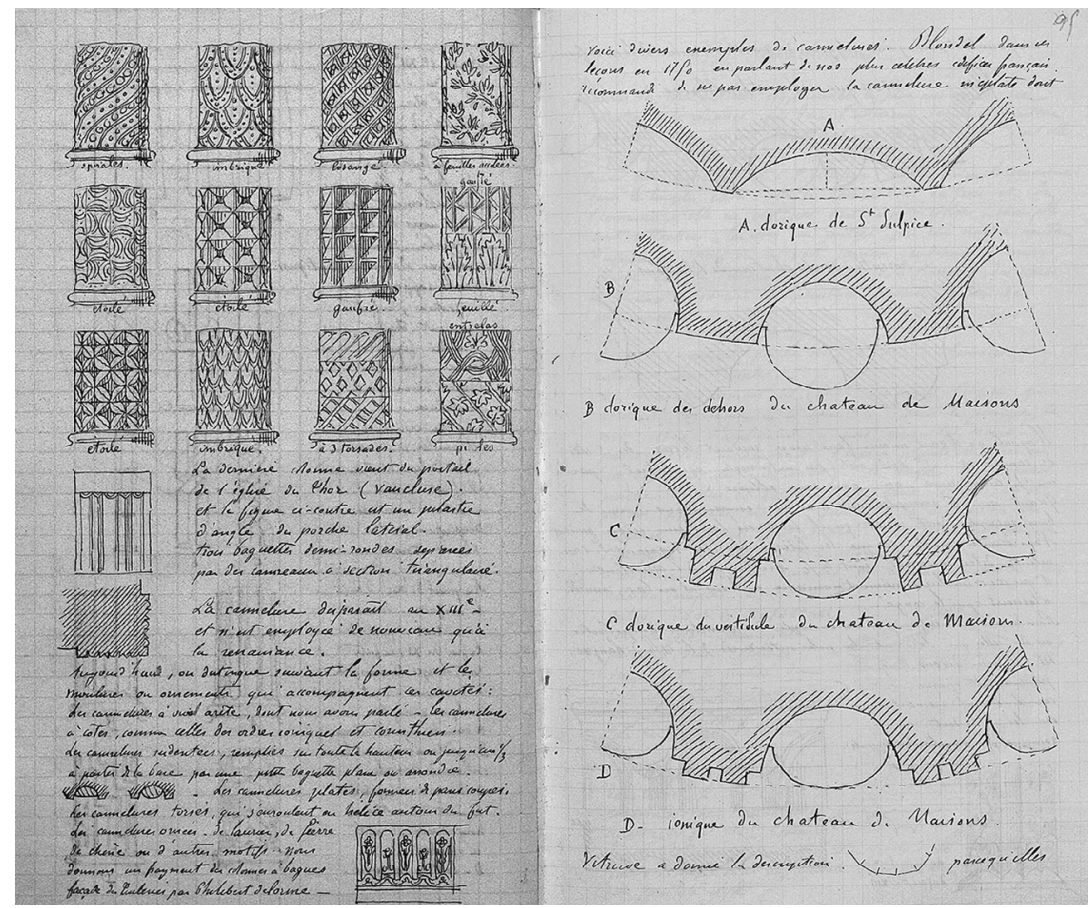

Ill. 3 : Alphonse Goutès, Cours d'architecture, vol. 1, Architecture, Éléments et principes, s.d., p. 94-95, Bibliothèque municipale de Montpellier, Fonds patrimoniaux, MS0244(1). (C) Médiathèque Centrale Émile Zola, Montpellier Méditerranée Métropole.

27. Ces cours ont lieu les mardi et jeudi de 16 à 17 h et accueillent trente-six élèves en 1887, sur les trois cents élèves réguliers de l'école. Arch. mun. de Montpellier, R1/9 (111).

28. Alphonse Goutès, Discours inaugural au cours d'histoire et de théorie [1878], dans Cours d'architecture, Bibliothèque municipale de Montpellier, Fonds patrimoniaux, MS0244 (13-8).

29. Nomination le 28 mai 1886. Ces cours ont lieu tous les jours de 6 à 8 h et de 17 à 19 h. Arch. mun. de Montpellier, R1/9 (107).

30. Alphonse Goutès, Cours d'architecture, Bibliothèque municipale de Montpellier, Fonds patrimoniaux, respectivement vol. 1 et 2 ( $1^{\text {re }}$ année) et 3 et 4 ( $2^{\mathrm{e}}$ année). 


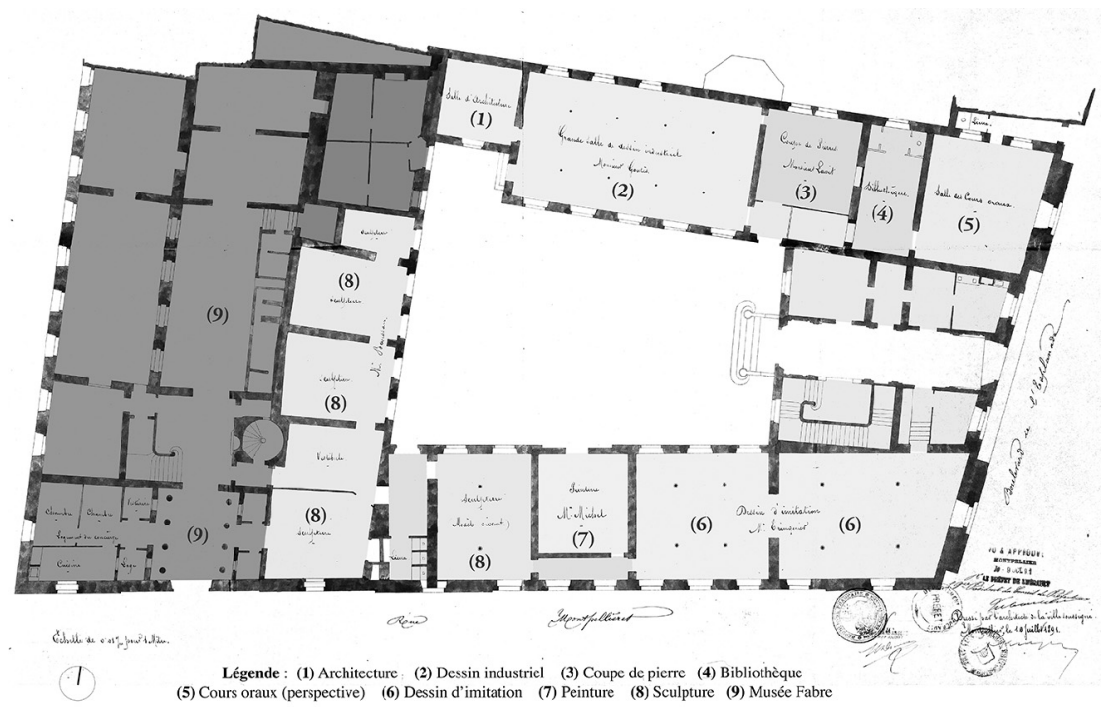

Ill. 4 : Alphonse Goutès, Cours d'architecture, vol. 3, $2^{\mathrm{e}}$ année, $1^{\mathrm{er}}$ volume. Construction, s.d., p. 240-241, Bibliothèque municipale de Montpellier, Fonds patrimoniaux, MS0244(3). (C) Médiathèque Centrale Émile Zola, Montpellier Méditerranée Métropole.

pour aborder l'année suivante les notions relatives à la construction, amenant progressivement les élèves à des exercices de composition ${ }^{31}$. L'approche pédagogique repose sur des leçons pratiques, qui sont complétées par des cours oraux et des démonstrations au tableau. En 1887, le cours accueille quatre-vingt-onze élèves et a lieu tous les jours. L'architecte assurera ce cours d'architecture pendant dix ans avant d'ouvrir un atelier proprement dit, puis un cours de "composition décorative ${ }^{32}$ conçu comme appliqué à l'industrie. Pour l'installation de ce nouveau cours, Goutès adresse une demande de renouvellement du matériel et réclame notamment l'achat d'un mégagraphe ${ }^{33}$. L'ensemble de ces cours a lieu dans les salles attenantes au musée Fabre et à l'Esplanade où, manquant d'espace et de lumière, ils bénéficient d'un réaménagement des locaux en $1893^{34}$ (ill. 4).

Un corpus monumental, miroir d'une circulation des savoirs

Ces manuscrits forment un ensemble de 25 volumes, dont certains composés de plusieurs tomes, pour un total de 38 carnets in $-8^{\circ}$, soit un ensemble d'environ 4700 pages de textes et dessins. Un premier aperçu fait d'emblée apparaître le caractère éclectique des sujets représentés, parmi lesquels se trouvent, au milieu des modèles antiques, médiévaux et renaissants, nombre d'édifices contemporains (ill. 5).

31. Idem, vol. 2 et 3.

32. Cours tenu en 1897 et 1898 . Arch. mun. de Montpellier, R1/9 (108) et M4/6.

33. "Appareil à rouleau qui permet de dessiner debout des motifs de plus de 3 mètres de haut ". Lettre d'A. Goutès au Directeur, 12 décembre 1896, Arch. mun. de Montpellier, M4/6.

34. Arch. mun. de Montpellier, M4/6. Voir également ADH 2O172/54, cf. ill. 4. 


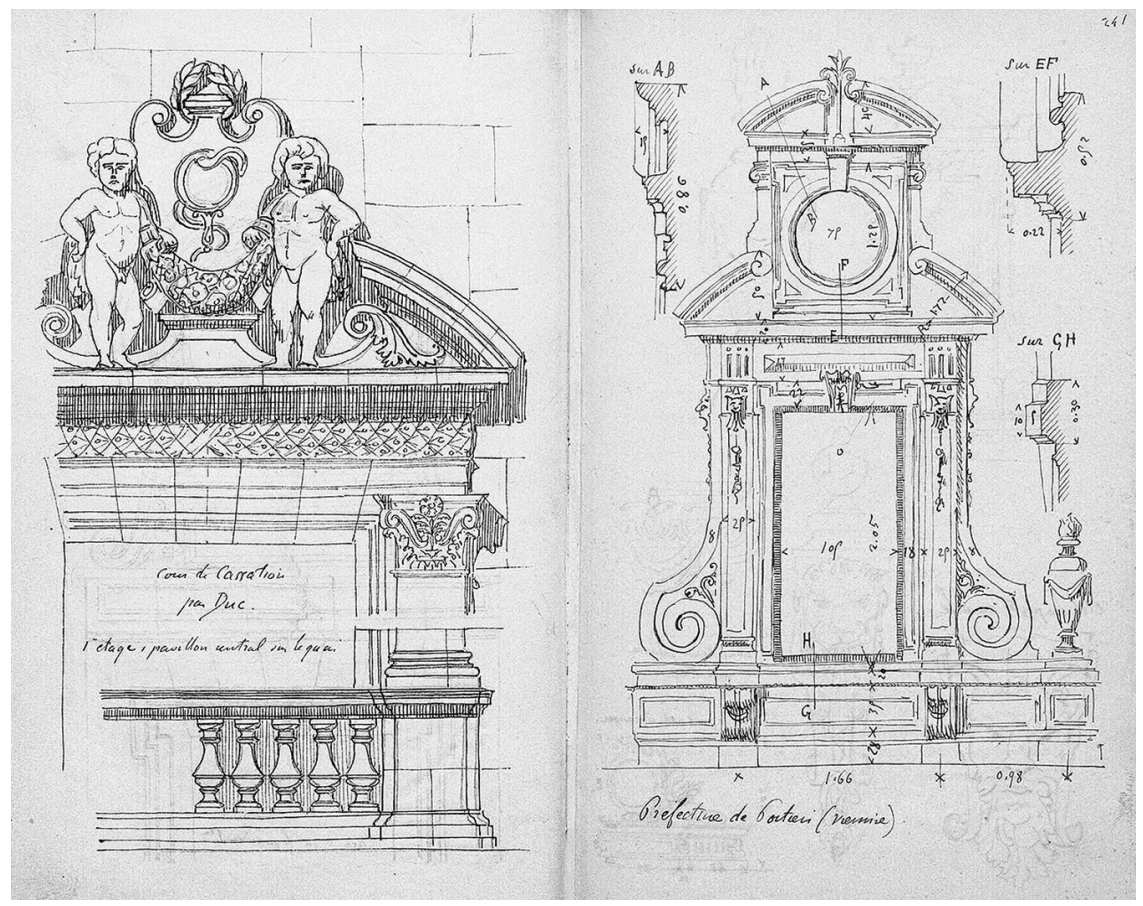

Ill. 5 : Antony Kruger, architecte de la ville de Montpellier, Plan de l'École régionale des beaux-arts de Montpellier, 10 juillet 1891, Archives départementales de l'Hérault 2 O 172 54. () Archives départementales de l'Hérault.

Ces modèles s'inspirent souvent de sources bien connues, telles que les ouvrages de Charles Blanc ${ }^{35}$ et César Daly ${ }^{36}$, mais aussi de Viollet-le-Duc ${ }^{37}$ et Jules Bourgoin ${ }^{38}$ dont il emprunte d'ailleurs les ouvrages à la bibliothèque de l'école ${ }^{39}$. Cet éclectisme stylistique, qui reflète bien la formation de Goutès à l'École des beaux-arts au sein de l'atelier Questel, peut être mieux compris à la lumière de ses références théoriques. Sur ce plan, l'architecte puise à des sources très variées, où se côtoient les traités traditionnels de théorie des ordres et des écrits, plus récents, d'ingénieurs comme Léonce Reynaud, Émile Trélat ou de l'ingénieur archéologue nîmois Auguste Aurès. En matière d'histoire de l'architecture, Goutès s'appuie principalement sur

35. Charles Blanc, Grammaire des arts du dessin, Paris, Vve Jules Renouard, 1867, 720 p.

36. César Daly, Motifs historiques d'architecture et de sculpture d'ornement pour la composition et la décoration extérieure des édifices publics et privés [...], 2 vol., in-fol., Paris, Morel, 1869.

37. Eugène Viollet-le-Duc, Réponse à $M$. Vitet, à propos de l'enseignement des arts $d u$ dessin, Paris, Morel, 1864 .

38. Jules Bourgoin, Grammaire élémentaire de l'ornement. Pour servir à l'histoire, à la théorie et la pratique des arts, et à l'enseignement, Paris, G. Delagrave, 1880, 207 p.

39. Bibliothèque de l'École des beaux-arts, registre de prêts (1881-1961), Arch. mun. de Montpellier, $2 \mathrm{R}$. 
sa lecture de Louis Batissier ${ }^{40}$ et de Viollet-le-Duc ${ }^{41}$, dont les œuvres ${ }^{42}$ sont justement adressées à l'école par Anatole de Baudot en juillet $1885^{43}$.

Mais la curiosité de Goutès s'étend bien au-delà de ces domaines ; aussi peut-on identifier des emprunts à divers ouvrages issus d'autres disciplines, comme la philosophie. Goutès s'avère ainsi un lecteur attentif de philosophes de son temps comme Edgar Quinet, Théodore Jouffroy, Charles Lévêque et Charles Blanc, dont l'influence, centrale dans le débat architectural, est bien connue ${ }^{44}$. Au-delà du contexte français, Goutès se réfere également aux pensées de Creuzer, de Hegel et, plus proche de lui, d'Herbert Spencer. Dans le sillage d'un débat philosophique attentif aux conditions de perception sensible et psychologique, cet intérêt pour l'esthétique s'élargit, chez ce professeur de perspective, à la psychologie ${ }^{45}$. Quelques décennies après les découvertes de Choisy relatives aux corrections optiques des temples grecs, Goutès se montre également attentif aux recherches en optique ${ }^{46}$. Dans les domaines scientifiques, sa curiosité ne se limite pas à des lectures spéculatives et peut bien sûr alimenter ses enseignements. Ses cours de composition décorative semblent ainsi nourris par son intérêt pour les processus de morphogénèse observés dans le monde animal et dans les éléments naturels ${ }^{47}$. Quant à ses cours de perspective et de géométrie descriptive, Goutès y recourt à la "méthode Adhemar " ${ }^{48}$. S'il est encore trop tôt pour distinguer une doctrine générale claire, on peut néanmoins faire l'hypothèse d'une influence significative des théories contemporaines de l'ornement et de celles de Viollet-le-Duc, dont Goutès semble retenir une lecture raisonnée de l'histoire de l'architecture au service du projet et l'idée d'un rationalisme structurel, que Goutès médite à travers le filtre des théories d'ingénieurs.

40. Goutès reproduit à la plume une gravure du pronaos du temple d'Isis à Philae, tirée de : L. Batissier, L'Histoire de l'art monumental, Paris, Furne, 1860 (1845), p. 112 ; Alphonse Goutès, op. cit., vol. 2, p. 91.

41. En particulier son Dictionnaire raisonné de l'architecture française du $X I^{e}$ au $X V I^{e}$ siècle, 10 vol., Paris, B. Bance, A. Morel, 1854-1868.

42. Eugène-Emmanuel Viollet-le-Duc, Compositions et dessins de Viollet-le-Duc, publiés sous le patronage du Comité de l'œuvre de maître, Paris, Librairie centrale d'architecture, 1884, 233 p.

43. Lettre d'Anatole de Baudot au Maire de Montpellier, juillet 1885, Arch. mun. de Montpellier, M4/6.

44. Estelle Thibault, La Géométrie des émotions. Les esthétiques scientifiques de l'architecture en France, 1860-1950, Wavre, Mardaga, 2010, notamment p. 25-58.

45. Sur ce plan, Goutès cite notamment la figure d'Albert Lemoine (1824-1874).

46. Alphonse Goutès, Cours d'architecture, Bibliothèque municipale de Montpellier, Fonds patrimoniaux, vol. 2 et 3. Goutès y fait référence aux physiciens Johann Karl Friedrich Zöllner (1834-1882) et Ogden Nicholas Rood (1831-1902).

47. Outre son intérêt pour les œuvres de Charles Darwin (1809-1882), Goutès s'intéresse aux travaux de Julius Bernstein (1839-1917) en physiologie, et de John Tyndall (1820-1893) en glaciologie.

48. Joseph-Alphonse Adhemar, Cours de mathématiques à l'usage de l'ingénieur civil, traité de géométrie descriptive, coupe des pierres, Paris, 1834 ; Traité de perspective, Paris, 1838, in- $8^{\circ}$ et 62 pl. in-folio ; et Traité des ombres. Théorie des teintes, des points brillants et de la perspective cavalière, Paris, Mathias, 1852. 


\section{Héritages pédagogiques et postérité d'Alphonse Goutès}

À la lumière de ces enseignements, le professorat d'Alphonse Goutès permet de prendre la mesure de l'ambitieuse refondation de l'École régionale des beaux-arts de Montpellier à la fin du XIX ${ }^{\mathrm{e}}$ siècle. Si les contenus des cours démontrent une attention aux débats théoriques et scientifiques de l'époque, l'approche pédagogique générale s'inscrit quant à elle dans le sillage des évolutions récentes des enseignements dispensés à Paris. Les cours de géométrie descriptive et d'esthétique, introduits à l'École des beaux-arts suite à la réforme de 1863, sont d'ailleurs assurés par Goutès à Montpellier. Plusieurs cours dispensés à Paris y font néanmoins défaut, comme les cours de physique ou de comptabilité. Si la diversification des cours ne peut alors égaler celle de l'établissement parisien, dont le programme s'enrichit encore en 1883, c'est qu'à Montpellier, la multiplication des disciplines enseignées n'entraîne pas avec elle celle du nombre d'enseignants. Ainsi, c'est bien à la curiosité et à la polyvalence d'un professeur comme Goutès, dispensant jusqu'à sept matières différentes, que l'on doit les progrès de la section d'architecture de l'École régionale de Montpellier dans les dernières décennies du XIX ${ }^{\mathrm{e}}$ siècle. Une telle évolution répond à l'ambition croissante de l'École régionale des beaux-arts de Montpellier, dans le contexte particulier du débat, qui s'affirme alors à l'échelle nationale, sur l'enseignement de l'architecture dans les villes régionales. À cet égard, il est significatif que Goutès s'implique au premier chef dans ce débat, étant avec son ami Léopold Carlier, les deux représentants montpelliérains signataires du vœu de Toulouse 49 .

Au cours de cette période se confirme avant tout la vocation hybride d'une école à la fois d'art et d'application, préparatoire et professionnelle. L'enseignement des trois arts permet aux jeunes artistes d'y faire mûrir leurs différents talents, passant parfois d'un art à l'autre : l'élève Henri Michel entre à l'école dans la section de peinture et en sort architecte, tandis que Louis Marcadier intègre la section d'architecture pour exercer comme peintre et sculpteur. La scolarité des élèves n'est donc pas toujours linéaire au sein de cette école polyvalente. Aussi, il paraît difficile d'estimer le niveau général des élèves qui semblent de talents très inégaux, les uns se destinant à une carrière d'ornemaniste ou d'artisan quand d'autres embrassent une ambition d'artiste et poursuivent une formation discontinue prolongée à Paris. Une dizaine d'élèves architectes de l'école connaîtront ainsi un parcours remarqué et pourront, grâce au soutien des bourses accordées par la ville de Montpellier ${ }^{50}$, achever leur formation à Paris.

49. Déclaration signée lors de l'assemblée générale de l'Association Provinciale en juin 1894, formalisant le souhait de voir toutes les sociétés régionales d'architectes consultées afin de former la commission d'étude et d'organisation sur l'enseignement, cf. Jean-Pierre Epron, op. cit., p. 51-58 et 233.

50. ADH 4T 24, Bourses départementales et municipales (1878-1931). 


\section{Les élèves d'Alphonse Goutès}

Parmi les sujets montpelliérains admis à l'École des beaux-arts, la majorité sera issue des élèves de Goutès. En l'espace d'une dizaine d'années, ceux-ci ${ }^{51}$ intègrent tour à tour l'école parisienne, où ils se distingueront par des médailles en dessin comme en histoire, ce qui suggère les bénéfices des enseignements reçus à Montpellier. Au-delà du cadre scolaire, l'héritage pédagogique d'Alphonse Goutès peut enfin s'apprécier à travers les trajectoires professionnelles de ses élèves. Après leur séjour parisien, ceux-ci essaimeront dans les départements du Midi, soulignant par là le rayonnement régional de l'enseignement de Goutès. Ses élèves y exerceront comme architecte pour l'édification de bâtiments civils et diocésains, mais également pour leur restauration, à l'image de Léon Vassas ${ }^{52}$, plus tard architecte ordinaire des Monuments historiques à Carcassonne. D'autres, enfin, s'engageront dans la voie de l'enseignement, comme Antoine Chevalier par la suite professeur à Sète, Henri Michel, et plus tard Gaston Brun, successeurs de Goutès comme professeur de géométrie à Montpellier, illustrant la longévité de son héritage pédagogique.

À l'aube du XX siècle, l'École régionale des beaux-arts de Montpellier a bien diversifié son programme pédagogique, se rapprochant du modèle parisien, sans pour autant cesser de répondre aux débouchés offerts à ses élèves. Qu'il s'agisse des arts décoratifs, des professions d'architecte ou de professeur de dessin, ces perspectives professionnelles restent intimement liées à un contexte local caractérisé par le tissu artisanal et industriel de la région. L'École de Montpellier préfigure bien en cela le rôle assigné aux écoles régionales d'architecture créées à partir de 1905, répondant alors au souhait unanime chez les architectes exerçant dans les départements de placer « l'enseignement de l'architecture à la portée des jeunes gens qui ne peuvent venir le chercher à Paris " ${ }^{53}$. La pédagogie de Goutès, en particulier, paraît satisfaire l'idée, exprimée plus tard par Julien Guadet, d'une décentralisation de l'enseignement sans décentralisation esthétique : adapter les enseignements aux particularités régionales, sans constituer pour autant d'écoles régionalistes, dans le souci de permettre aux meilleurs élèves d'achever leurs études à Paris.

Dans le sillage des nombreux travaux menés sur l'histoire des enseignements ${ }^{54}$ et de l'édition architecturale ${ }^{55}$ au XIX ${ }^{\mathrm{e}}$ siècle, l'étude de l'enseignement montpellié-

51. Léon Vassas (1870-1948), Joseph Chevalier (1864-?) et Henri Michel (1866-?), seront tous les trois admis en 1889, Louis Carlier (1872-1955) et Paul Harant (1873-1953) en 1892, et Louis Marcadier (1874-?) en 1896. Arch. nat., dossiers d'élèves. Les élèves L. Vassas, J. Chevalier, L. Carlier et P. Harant intégreront l'atelier de Léon Ginain (1825-1898).

52. Léon Vassas sera le père de Robert Vassas (1909-2000), architecte en chef des Monuments historiques.

53. Julien Guadet, "Rapport présenté au nom de la commission instituée à l'effet d'étudier l'organisation d'écoles régionales d'architecture, par M. J. Guadet, inspecteur général des bâtiments civils, professeur à l'École nationale des beaux-arts ", Journal Officiel de la République française, 31 janvier 1903.

54. Citons, entre autres travaux, ceux d'Antoine Picon, Frédéric Seitz, John Garleff, Jean-François Belhoste, J.-Y. Dupont, Simone. Talenti, Jean-Philippe Garric, Estelle Thibault, Guy Lambert, Marie-Laure Crosnier Leconte et dernièrement, d'Amandine Diener.

55. Sur cette période, mentionnons ici en particulier les recherches de Jean-Philippe Garric, Marc Saboya et Béatrice Bouvier. 
rain soulève ainsi de multiples enjeux. Dotée d'un statut hybride d'école préparatoire pour les uns, et professionnelle pour les autres, l'école montpelliéraine occupe en somme une position ambivalente par rapport au contexte parisien : à la fois caisse de résonance, en aval des débats parisiens, et laboratoire d'expériences pédagogiques originales, en amont des formations achevées à Paris. De sorte que cette histoire, qui contribue à la connaissance d'un contexte régional particulier, met du même coup en lumière les conditions d'une circulation des savoirs, entre Paris et le Sud de la France. Tels sont bien les enjeux d'un décentrement de l'attention ${ }^{56}$ qui, déjà fructueuse en ce qui concerne les établissements parisiens ${ }^{57}$, invite à mettre au jour les transferts entre Paris et contextes régionaux, ainsi qu'aux marges de la profession, les relations entre architectes et artistes, artisans et entrepreneurs.

En 1898, la carrière de Goutès s'interrompt brutalement par une mort prématurée, à 59 ans. L'école de Montpellier connaît à ce moment une mutation profonde, causée par la conjonction de quatre départs à la retraite entre 1901 et $1905^{58}$, occasionnant un renouvellement presque complet du corps enseignant en quelques années. Ainsi, au moment même où l'implantation des Écoles régionales d'architecture est décidée, les cours de la section d'architecture voient décliner aussi bien leurs effectifs que leurs résultats ${ }^{59}$. On perçoit donc, enfin, les atouts et les faiblesses de l'établissement montpelliérain à l'aube du $\mathrm{XX}^{\mathrm{e}}$ siècle, et les raisons de l'occasion manquée de 1903. L'école de Montpellier privilégiera désormais les arts décoratifs ${ }^{60}$ au détriment de l'enseignement simultané des trois arts qui avait fait la réussite de l'école sous la direction d'Ernest Michel et le professorat d'Alphonse Goutès.

Théodore GUUINIC Architecte DE HMONP

Doctorant en Architecture à l'université Paul Valéry-Montpellier III Maître de conférences associé à l'ENSA de Montpellier

56. Citons dans cette démarche les recherches récemment engagées dans le cadre du programme HEnsA20.

57. Parmi ces nombreux travaux, citons à titre d'exemple Guy Lambert et Estelle Thibault (dir.), L'Atelier et l'amphithéâtre. Les écoles de l'architecture, entre théorie et pratique, Wavre, Mardaga, 2012, 217 p.

58. Départs d'Ernest Michel, directeur et professeur de peinture, en 1901, d'A. Baussan, professeur de sculpture, en 1901 de F. Lavit, professeur de stéréotomie en 1905, et d'A. Trinquier, professeur de dessin en 1905.

59. Sur ce point, voir D. Laredo, op. cit., p. 111.

60. Arch. mun. de Montpellier R1/9 (110) et R1/9 (107) ; voir également Arch. dép. de l'Hérault $4 \mathrm{~T} 23$. 